\title{
A Statistical Study of Telugu Treebanks
}

PRAVEEN GATLA

\section{Abstract}

The paper is an attempt to compare Hyderabad Telugu Treebank (HTTB) and HCU-IIIT-H Telugu Treebank from a statistical point of view. HTTB has 2,715 annotated sentences and HCU-IIIT-H TTB has 3,222 annotated sentences. Both the Treebanks were annotated by following Paninian Grammar Formalism proposed by Bharati, A.; Sharma, D.M.; Husain, S.; Bai, L.; Begam, R. and Sangal, R. (2009). HTTB is an interchunk-based treebank data. HCU-IIIT-H TTB is an intrachunk-based treebank data. Both the treebanks' data size is random. Later, the paper discusses the Telugu Treebanks in detail. The paper focuses on statistical frequencies viz. POS, Chunk and Syntactic labels. VM (3807 times) and NN (5486 times) are the frequent POS labels in HTTB and HCU-IIIT-H $T T B$ respectively. NP (7954 and 6223 times) is the frequent phrasal category in both the treebanks. The most frequent $k$ labels are kartāa $(k 1)$ (2375-2381 times) and karma(k2) (14081437 times) and non-frequent label is karana(k3) (17-39 times) in both the treebanks. The most frequent non-k-labels are verb modifier (vmod) (949 times) and noun modifier (nmod) (1033 times) in both the treebanks. The statistical distribution mentions the coverage of the labels (kāraka, non-kāraka) of both the Telugu treebanks. Later it discusses the comparison of both the treebanks and tries to provide the reasons for the highest and lowest frequencies in both the treebanks. $k 1$ and $k 2$ have $60 \%$ of the coverage in karaka labels, vmod, nmod, adv, ccof, pof also has $60 \%$ of the coverage in non-karaka labels. This kind of statistical study can help to boost the accuracy of the parser.

Keywords: Treebank, Paninian Grammar, Telugu, kāraka, non-kāraka, Statistical Frequency, Coverage, Parser. 
Praveen Gatla

\section{Introduction}

The creation of language resources is one of the most challenging tasks in the field of Natural Language Processing. One needs to read and understand the natural language text by making use of one's intuition as a native speaker and his linguistic knowledge. It requires a lot of training in the field of language and linguistics to encode linguistic information. Based on that, treebanks can be created for Indian languages. A plain or simple text, which is encoded with linguistic information, is called annotated data. This kind of lexical resource is useful to develop syntactic parsers for Indian languages. Such tasks involve huge human resources, time, and financial support. In the past, treebanks were created for English and other languages based on different grammatical formalisms (Phrase Structure Grammar, Dependency Grammar, Paninian Grammar, Context-Free Grammar, Universal Dependency Grammar) which are Penn Treebank (Marcus, M.; Santorini, B. \& Marcinkiewicz, M. A. 1993), Prague Dependency Treebank (Hajičová 1998) so on. Each Grammar Formalism has its own limitations. In order to create treebanks, researchers have used Paninian Grammar, Dependency Grammar, and Universal Grammar Formalisms, which have helped to create syntactic parsers for Indian languages (Hindi, Telugu, Tamil, Marathi, Bangla). The main goal of the present research paper is to compare the two Telugu treebanks. They are HTTB ${ }^{1}$ (Praveen 2019) and HCU-IIIT-H TTB $^{2}$ (Nallani, S.; Shrivastava, M; \& Sharma, D. 2020). Praveen (2019) has created 2,715 (sentences) Telugu treebank data by following Paninian Grammar Formalism. Apart from this, HCU-IIIT-H has developed 3,222 Telugu treebank data (sentences) based on Paninian Grammar Formalism. We

\footnotetext{
${ }^{1}$ Hyderabad Telugu Treebank.

${ }^{2}$ HCU-IIIT-H Telugu Treebank.
} 
considered these two Telugu treebanks for the statistical study. In this paper, we compare both the treebanks from the statistical point of view and try to identify the average number of words per sentence, statistical frequency of Parts of Speech (POS) categories, statistical frequency of Chunks (Phrases), statistical frequency of Telugu treebanks data (kāraka labels and non-käraka labels). The paper is organized into five sections. Section 2 discusses related works on treebanks (Indian Languages). Section 3 presents a brief overview of Telugu treebanks, Section 4 describes the statistical frequency of kāraka and non-kāraka labels of Telugu treebanks, the significance of the kāraka and non-käraka labels in both the Telugu treebanks. Finally, we conclude our paper in Section 5.

\section{Related Works}

In this section, we discuss some of the relevant research works on treebanks. Treebanks have been developed by following different grammar formalisms. They are Phrase Structure Grammar, Paninian Grammar, Context-Free Grammar, Dependency Grammar, Universal Dependency Grammar. Marcus (1993) describes the construction of a large annotated corpus which is named as Penn Treebank. This resource was developed as a part of the Penn Treebank Project. It was a three-year project from 1989 to 1992. This corpus consists of POS information and skeletal syntactic structure (partially). Penn Treebank is a good resource for linguistic theory (Robert Ingria) and psychological modeling (Niv 1991). Penn Treebank has been extended to other languages like Chinese, Arabic, French, Spanish, etc. Begum, R.; Husain, S.; Bai, L. and Sharma, D. M. (2008) made an attempt to create Hindi annotated data using the Paninian Grammatical model for the first time. They have annotated almost a million words (nearly 1403 sentences) of Hindi corpus. In this framework, twentyeight relations were considered for the annotation. It consists 
of six basic kāraka's. They are adhikarana (k7) 'location', apaadaan (k5) 'source', sampradaan (k4) 'recipient', karaṇa (k3) 'instrument', karma (k2) 'theme', kartā (k1) 'agent'. Bharati, A.; Gupta, M., Yadav, V., Gali, K., and Sharma, D. M. (2009) proposed a simple parser for Indian languages in a dependency framework. They describe a syntactic parser, which follows a grammar-driven approach. They described a grammar-oriented model that makes use of linguistic features to identify relations.

The proposed parser was modeled based on the Paninian grammatical approach. They have shown that with the help of robust rules one can achieve high performance in the identification of various levels of dependency relations. Bhatt, R.; Narasimhan, B.; Palmer, M.; Rambow, O.; Sharma, D.M. and Xia, F. (2009) discusses multi-representational and multilayered treebank. They discuss the multi-representational treebank which provides clues for syntactic dependency version and phrase structure version based on the DS (Dependency Structure) and PS (Phrase-Structure) guidelines. They have developed this treebank based on PropBank and predicate-argument annotation. This approach anticipates that the addition of the PropBank annotation to Dependency Structure (DS) will provide a rich and adequate amount of structure for PS conversion. De, S.; Dhar, A. and Garain, U. (2009) have worked on Bangla parsing by following constraint-based dependency parsing. They have used 1000 Bangla annotated sentences to train the system. Chatterji, S.; Sonare, P.; Sarkar, S and Roy, D. (2009) proposed a hybridbased approach to parse Bengali sentences. The system tried to work on data-driven dependency parsers. Shailaja (2009) has developed simple Sanskrit sentences rule-based parser. The CLIPS expert system was used to formulate the rules. The developed parser can handle käraka and upapada vibhakti relations. Fifteen rules were formulated to handle different 
types of Sanskrit sentences. This attempt was the preliminary attempt to develop the Sanskrit parser. Kulakarni (2010) has made a formal attempt to explore the kāraka relations in Sanskrit by using Paninian Grammar Formalism. The main attempt is to identify the various kâraka relations between the words to extract only syntactic-semantic relations which depend on linguistic or grammatical information in a sentence.

As a part of it, they have annotated 110 (525 tokens) simple sentences which have a single finite verb. The average length of the sentence is 5 words and the maximum length of the sentence is 14 words. Among 110 sentences, 97 sentences output was correct and the remaining 13 sentences were wrongly parsed. Kulakarni and Ramakrishnamacharyulu (2013) discussed some of the specific issues in parsing the Sanskrit texts. In this work, they tried to handle different kinds of constructions in Sanskrit. They are abhihita, indeclinables (avyaya), inter-sentential connectives, anaphora, conjunctions, and disjunctions. Gade (2014) has worked out on two different treebanks' (Hindi and Sanskrit). She has considered 2300 sentences manually and extracted 1800 sentences which are released for the ICON-2009 Tool Contest (Hindi and Sanskrit) (Husain, S.; Mannem, P.; Ambati, B.R. and Gadde, P. 2010). Vempaty, C.; Naidu, V.; Husain, S.; Kiran, R.; Bai, L.; Sharma, D.M., and Sangal, R.; (2010) were the first attempt to create Telugu Treebank at LTRC, IIIT- $\mathrm{H}^{3}$. They manually annotated 1457 Telugu sentences by following Paninian Grammar Formalism (Bharati, A.; Sharma, D.M.; Husain, S.; Bai, L.; Begam, R. and Sangal, R. 2009). Later as a part of ILIL $\mathrm{MT}^{4}$ project (Phase II) funded by the Ministry of Electronics and Information Technology (MeitY) $)^{5}$,

\footnotetext{
3 Language Technology Research Center, International Institute of Information Technology, Hyderabad.

${ }_{5}^{4}$ Indian Languages to Indian Languages Machine Translation Systems

${ }^{5}$ https://meity.gov.in/
} 
Government of India, it was decided to develop a simple syntactic parser for the nine Indian languages. As a part of this task, a group led by Umamaheshwar Rao (2010-2016) have developed 5,000 (sentences) HCU Telugu treebank at CALTS, $\mathrm{UoH}^{6}$.

Recently, by combining IIIT-H Telugu treebank consisting of 1600 sentences from ICON 2009 tools contest, 200 (sentences) Telugu treebank data (IIIT-H), and 5,000 (sentences) HCU Telugu Treebank (Umamaheshwar Rao, G.; Koppaka, R.; Addanki, S. 2012; Rajyarama \& Srinivas, 2015a \& 2015b) have been combined into one set. Nallani, S.; Shrivastava, M, and Sharma, D.M. (2020) have formatted, cleaned and released the licensed final Telugu treebank data consisting of 3,222 sentences under the Creative Commons License Attribution Noncomercial Share 4.0.1. International. International. Rama and Soumya (2017) have worked on Telugu treebank. They have followed the Universal Dependency framework and annotated 1328 sentences from Telugu grammar. The treebank developed by them is freely available at Universal Dependencies ${ }^{7}$ version 2.1. They discussed corpus annotation, parts-of-speech annotation, morphology, Universal Dependency relations in their paper. They also reported the preliminary tagging and parsing results with UDPipe. Apart from that, Universal Dependency treebanks have been developed for nine Indian languages. They are Bhojpuri, Hindi, Hindi-English, Kangri, Magahi, Marathi, Sanskrit, Tamil, and Urdu. There are four upcoming Indian languages under UD treebank. They are Bengali, Assamese, Kannada, Pnar.

${ }^{6}$ Centre for Applied Linguistics and Translation Studies, University of Hyderabad.

${ }^{7}$ https://github.com/UniversalDependencies/UD_Telugu-MTG

8https://universaldependencies.org/ 


\section{Telugu Treebanks}

Telugu is a Dravidian language. It is a morphologically rich language. A series of suffixes can be attached to a single root in Telugu. In the development of the treebanks, we give much more importance to the morphological (inflection) information because it gives gender, number, person, case information for nouns, tense, aspect, modality information for verbs. All these interpretations reflect at the morphological level in Telugu. Here, we consider two Telugu treebanks which are developed based on Paninian Grammar Formalism for statistical study. Based on this formalism, dependency structure (DS) guidelines were developed by Akshar Bharati group ${ }^{9}$. As a part of IL-IL MT project ${ }^{10}$, this group developed the annotation guidelines to create treebanks for Indian languages. The baseline for creating these guidelines is Paninian grammar. In this framework $^{11}$, a sentence is considered as one unit where the verb is the central notion. Apart from that, other constituents also play an important role in a sentence. The käraka relations denote syntactico-semantic relations between the verb and other constituents in a sentence (Cf. Sangal, R.; Chaitanya, V. $\&$ Bharati, A. 1995). There are two types of relations in this scheme i.e., kāraka and non-kāraka. The kāraka relations are kartā (k1) 'doer', karma (k2) 'object', karana (k3) 'instrument', sampradāna (k4) 'receiver', apādāna (k5) 'source', adhikaraṇa (k7) 'location' and non-kāraka relations are șașthī (r6) (genitive, possessive), hètuh (rh) 'reason', tādarthya (rt) 'purpose', adjectival modifiers (jjmod) and adverbial modifiers (rbmod) etc. Based on the types of relations, the

\footnotetext{
9 AnnCorra: Tree Banks for Indian Languages Guidelines for Annotating Hindi Treebank (Ver 2.0).

${ }^{10}$ Indian Languages to Indian Languages Machine Translation System Project (Phase I and II) funded by Ministry of Electronics and Information Technology, Government of India.

${ }^{11}$ AnnCorra: TreeBanks for Indian Languages.
} 
dependency tags are also classified into two types. They are inter-chunk ${ }^{12}$, intra-chunk ${ }^{13}$. The chunks (quasi phrases) are considered as the heads in inter-chunk relations (annotation), whereas in intra-chunk annotation each word or token is marked with a relation. Bharati, A., Sharma, D.M., Husain, S., Bai, L., Begam, R., and Sangal, R. (2009) have developed DS guidelines to create treebanks. In this framework, käraka and non-kāraka relations are denoted in a sentence. käraka relations have tags that start with a ' $k$ ' and are followed by a numerical digit (e.g., 1 to 5 and 7). They are kartā (k1), karma (k2), karana (k3), sampradāna (k4), apādāna (k5), adhikaraṇa (k7), etc. These kāraka's are further fine-grained as sub-tags of kartā such as kartā samānādhikaraṇa (k1s), prayojya kartā (Causee; jk1), prayojaka kartā (Causer; pk1). The non-kāraka tags either begin with ' $r$ ' or ' $c$ ' or ' $p$ '. They are șașthī 'genitive or possessive' (r6), hētuh (reason) (rh), tādarthya 'purpose' (rt), Coordination (ccof), Part of (pof), etc. The different types of dependency relations are mentioned in Bharati, A.; Sharma, D.M.; Husain, S.; Bai, L.; Begam, R. and Sangal, R. (2009) which shows "the relations from coarser level to finer level on a modifier-modified paradigm" (Bharati, A.; Sharma, D.M.; Husain, S.; Bai, L.; Begam, R. and Sangal, R. 2009).

\subsection{Hyderabad Telugu Treebank (HTTB)}

We have adopted DS Guidelines which are developed by Bharati, A.; Sharma, D.M.; Husain, S.; Bai, L.; Begam, R. and Sangal, R. (2009) which are followed to create HTTB. As a part of it, we have developed 2,715 Telugu treebank data by following DS guidelines in 2009. Sentences are extracted from various sources such as literary texts and grammar books viz. Krishnamurti and Sarma (1968), Krishnamurti and Gwynn

${ }^{12}$ Inter-chunk mark the kāraka relations that occurs between any two chunks.

${ }^{13}$ tags mark the kāraka relations within a chunk. 
(1985), Krishnamurti (1991, 2003, 2009), Ramarao (2002, 1975), Subrahmanyam (1984), Ramakrishna Reddy (1986), Subbarao (2012), Usha Rani (1980), Prakasam (2018) to develop the Telugu Treebank data. In this treebank, we have considered Bharati, A.; Sangal, R.; Sharma, D.M. and Bai, L. (2006) POS categories. This tagset consists of 26 POS categories. It is an inter-chunk-based treebank data.

\subsection{HCU-IIIT-H Telugu Treebank (HCU-IIIT-H TB)}

Nallani, S.; Shrivastava, M and Sharma, D. (2020) combined the HCU TTB and IIIT-H TTB data into one set and made it available for public access. In this, there are 3,222 annotated sentences. It is intra-chunk-based data. Telugu POS tagged data have been converted by following the latest BIS tagset ${ }^{14}$ (Bureau for Indian Standards). The BIS tagset is a standardized POS tagging guideline for all Indian languages. This tagset consists of 11 POS categories and most of the categories have further divided into fine-grained POS tags.

\section{Statistical Frequency of Telugu Treebanks}

In this section, we discuss the statistical frequencies of HTTB and HCU-IIIT-H TTB. The average length of the sentences of HTTB (Cf. Praveen, 2019) and HCU-IIIT-H TTB (Cf. Nallani, S.; Shrivastava, M, and Sharma, D. 2020) are 6 and 5.5 respectively. As a part of this exercise, we have listed out the frequencies of POS categories, phrases (chunks), käraka, and non-kāraka labels which are discussed in this section.

\subsection{Statistical Frequency of Parts of Speech (POS) Categories of Telugu Treebanks}

We have identified the occurrences of each POS category in the Telugu treebanks dataset. Here, we have considered only those POS categories which were used in Bharati, A.; Sangal,

\footnotetext{
${ }^{14}$ http://tdil-dc.in/tdildcMain/articles/134692Draft\%20POS\%20Tag\%20standard.pdf
} 
Praveen Gatla

R.; Sharma, D.M. and Bai, L. (2006) for calculating statistical frequency. In HTTB, VM (Main Verb) has occurred 3,807 times (highest) and QO(Ordinal) has occurred 11 times (lowest). In HCU-IIIT-H TTB, NN (Common Noun) has occurred 5486 times (highest) and PSP (Post-position) has occurred 2 times (lowest). In the latest BIS tagset, Nallani, S.; Shrivastava, M and Sharma, D. (2020) have used RD_PUNC for $S Y M, P R \_P R Q$ for $W Q$ so and so forth. $R D P$ was not found in HCU-IIIT-H TTB. Parts of Speech (POS) categories and their frequencies are shown in Table 1. Hyphen denotes not found in Table1.

\begin{tabular}{|l|l|l|l|}
\hline $\begin{array}{l}\text { Sl. } \\
\text { No. }\end{array}$ & POS Tags & $\begin{array}{l}\text { HTTB Frequency } \\
\text { Count }\end{array}$ & $\begin{array}{l}\text { HCU-IIIT-H } \\
\text { TTB Frequency } \\
\text { Count }\end{array}$ \\
\hline 1 & VM & 3807 & 4317 \\
\hline 2 & NN & 3509 & 5486 \\
\hline 3 & SYM/RD_PUNC & 2937 & 3330 \\
\hline 4 & PRP & 1741 & 1246 \\
\hline 5 & NNP & 1020 & 695 \\
\hline 6 & NST & 426 & 316 \\
\hline 7 & RB & 292 & 432 \\
\hline 8 & DEM & 261 & 237 \\
\hline 9 & WQ/PR_PRQ & 246 & 215 \\
\hline 10 & JJ & 230 & 414 \\
\hline 11 & VAUX & 175 & 59 \\
\hline 12 & CC/CC_CCS/CC_CCD & 164 & 262 \\
\hline 13 & QC & 125 & 274 \\
\hline 14 & PSP & 101 & 2 \\
\hline 15 & RP/RPD & 98 & 65 \\
\hline 16 & QF & 81 & 191 \\
\hline 17 & UT/CC_CCS_UT & 81 & 105 \\
\hline 18 & INTF & 46 & 85 \\
\hline 19 & RDP & 37 & - \\
\hline 20 & NULL & 19 & 19 \\
\hline 21 & CL & 13 & 14 \\
\hline 22 & QO & 11 & 21 \\
\hline
\end{tabular}

Table 1: POS Frequency in the Telugu treebanks 


\subsection{Statistical Frequency of Chunks (Phrases) of Telugu Treebanks}

We have identified the occurrences of each Phrasal (Chunks) category in Telugu treebanks dataset. We counted the frequency of each Phrasal category and their frequency in Table 2. Because HCU-IIIT-H TTB is available in the intrachunk format. We considered only chunk(phrasal) heads for the frequency count. Hyphen denotes not found in Table2.

\begin{tabular}{|l|l|l|l|}
\hline $\begin{array}{l}\text { Sl. } \\
\text { No. }\end{array}$ & $\begin{array}{l}\text { Phrasal } \\
\text { Categories }\end{array}$ & $\begin{array}{l}\text { HTTB Frequency } \\
\text { Count }\end{array}$ & $\begin{array}{l}\text { HCU-IIIT TTB } \\
\text { Frequency } \\
\text { Count }\end{array}$ \\
\hline 1 & NP & 6223 & 7954 \\
\hline 2 & VGF & 3739 & 3314 \\
\hline 3 & VGNF & 997 & 865 \\
\hline 4 & RBP & 170 & 458 \\
\hline 5 & VGNN & 124 & 126 \\
\hline 6 & BLK & 103 & 200 \\
\hline 7 & CCP & 162 & 317 \\
\hline 8 & JJP & 7 & 103 \\
\hline 9 & VGINF & - & 6 \\
\hline
\end{tabular}

Table 2: Chunk frequency in the Telugu treebanks

Noun Phrase has occurred 7954 and 6223 times in the HTTB and HCU-IIIT-H TTB respectively. It is the highest frequent phrasal category in both the treebanks. The lowest frequent phrasal category is JJ (Adjectival Phrase), VGINF (Infinitival Verbal Phrase) have occurred 7 and 6 times in HTTB and HCU-IIIT-H TTB respectively.

\subsection{Statistical Frequency of Telugu Treebanks (Labels)}

We have considered both the Telugu treebanks ${ }^{15}$. By using this annotated data, we have calculated the k-labels and non-klabels statistical frequency separately and discussed them in detail.

${ }^{15}$ HTTB and HCU-IIIT-H TTB 
Praveen Gatla

\subsubsection{Statistical Frequency of Hyderabad Telugu Treebank}

In HTTB, the highest and lowest frequency for kāraka labels are $k a r t a ̄(\mathrm{k} 1)$ käraka 2375, karaṇa $(\mathrm{k} 3)$ käraka 17 times respectively. Similarly, the highest and lowest frequency for non-kāraka labels are Verb Modifier (vmod) 949 times and associative (ras-k2) 4 times respectively. Figure 1 and 2 represents the statistical frequency of HTTB viz. kāraka labels and non-kāraka labels separately.

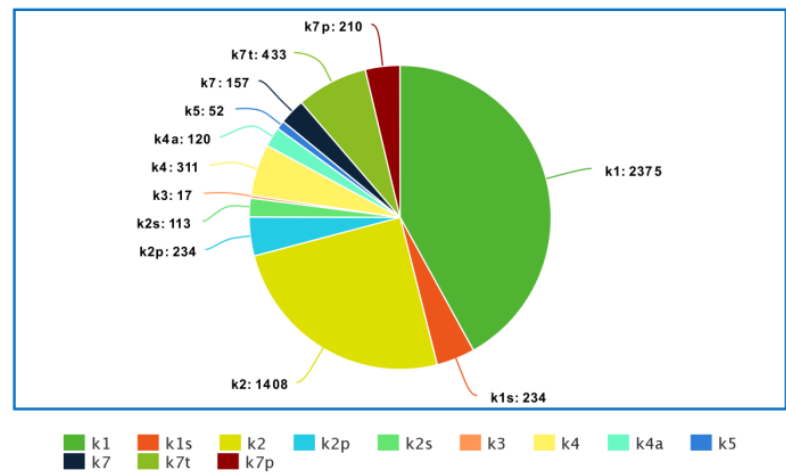

Figure 1: Statistical frequency of kāraka labels of HTTB

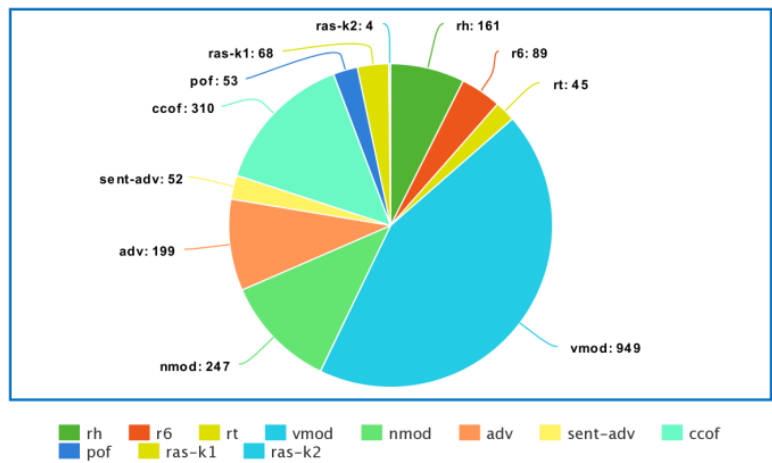

Figure 2: Statistical frequency of non-kāraka labels of HTTB 


\subsubsection{Statistical Frequency of HCU-IIIT-H Telugu Treebank}

In HCU-IIIT-H TTB, the highest and lowest frequency for kāraka labels are kartā $(\mathrm{k} 1)$ 2381, karma samānādhikarana (k2s) 32 times respectively. Similarly, the highest and lowest frequency for non-kāraka labels is Noun Modifier (nmod) 1033, ras-k2 (Relation for Associative) 6 times. Figure 3 and 4 represents the statistical frequency of HCU-IIIT-H TTB viz. käraka and non-käraka labels separately.

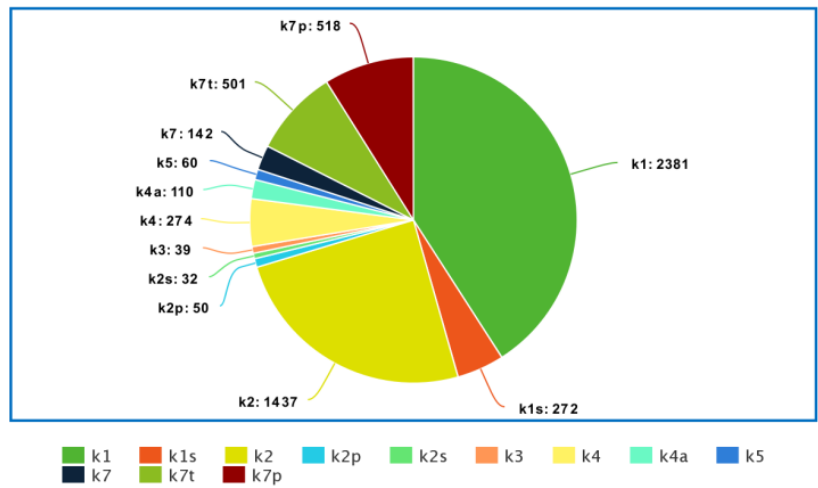

Figure 3: Statistical frequency of kāraka labels of HCU-IIIT-H TTB

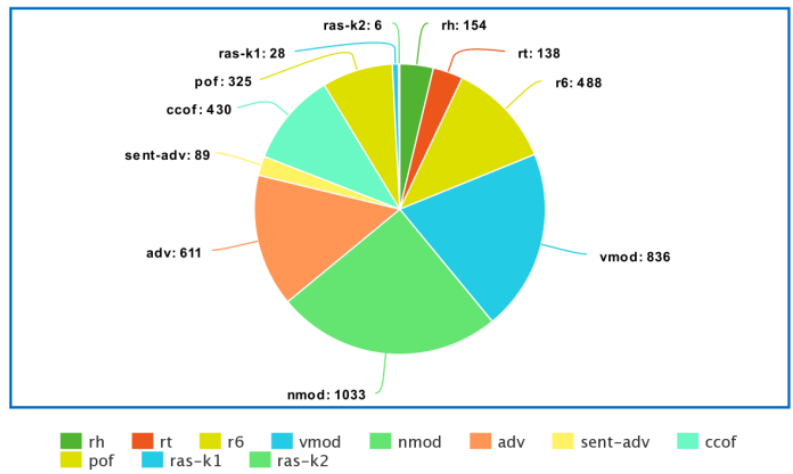

Figure 4: Statistical frequency of non-kāraka labels of HCU-IIIT-H TTB 


\subsection{Statistical Comparison of HTTB and HCU-IIIT-H TTB} Here, we try to compare both the Telugu treebanks statistics one by one based on the statistical frequency. The comparison of both the treebanks can be seen in Figure 5. We try to draw our observations based on the highest and lowest frequency of kāraka and non-kāraka labels. Here, one more important point is that the size of the Telugu treebanks is not the same. They are random in size. Statistical frequency of both the treebanks (HTTB and HCU-IIIT-H TTB) viz. k1 label is 2375-2381, k1s is $234-272, \mathrm{k} 2$ is $1408-1437, \mathrm{k} 2 \mathrm{p}$ is $234-50, \mathrm{k} 2 \mathrm{~s}$ is $113-32, \mathrm{k} 3$ is $17-39, \mathrm{k} 4$ is $311-274, \mathrm{k} 4 \mathrm{a}$ is $120-110, \mathrm{k} 5$ is $52-60, \mathrm{k} 7$ is $157-$ $142, \mathrm{k} 7 \mathrm{t}$ is $433-501, \mathrm{k} 7 \mathrm{p}$ is $210-518$, rh is $161-154$, r6 is $89-$ 488 , $\mathrm{rt}$ is $45-138$, vmod is $949-836$, nmod is $247-1033$, adv is $199-611$, sent-adv is $52-89$, pof is $53-325$, ccof is $310-430$, ras$\mathrm{k} 1$ is 68-28, ras-k2 is 4-6 etc ${ }^{16}$ (See Figure 1, 2, 3 and 4). Based on the two Telugu treebanks, one can predict that most of the time a sentence would have $\operatorname{karta}(\mathrm{k} 1), \operatorname{karma}(\mathrm{k} 2)$ for sure. It means that the subject and object are mandatory in a sentence. Another observation is that sampradāna(k4), apādāna(k5), adhikarana $(\mathrm{k} 7)$ are expected in a sentence optionally. In comparison with kartā(kl) and karma(k2), their $(k 4, k 5, k 7)$ occurrences or frequencies are very less in both the treebanks. When we compare non-kāraka relations or labels viz. hētu( $r h)$, verb modifiers (vmod), noun modifiers (nmod), coordination (ccof), sent-adv (Sentential Adverbs) frequencies are higher than tādarthya(rt), sașthī(r6), adverbs(adv), pof (Part of), rask1 (Associative with kartā), ras-k2 (Associative with karma). There are certain labels, which have a drastic difference in the frequency count. For example, $r 6$ is $89-488, r t$ is $45-138$, nmod 247-1033, adv 199-611, pof 53-325, ras-k1 68-28. HTTB data has a low frequency (treebank labels) in comparison with HCU-IIIT-HTTB.

\footnotetext{
${ }^{16}$ These numbers denote number of occurrences in HTTB and HCU-IIIT-H Telugu treebanks.
} 
A Statistical Study of Telugu Treebanks

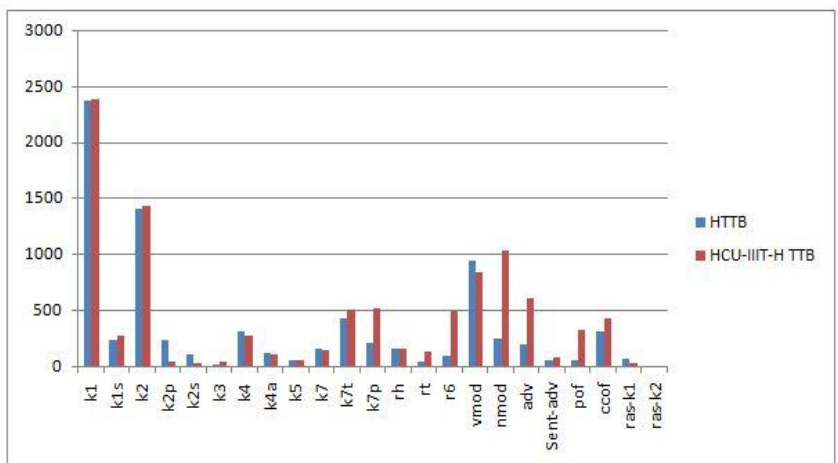

Figure

5: Comparison of HTTB and HCU-IIIT-H TTB labels (käraka and nonkāraka labels)

\subsection{Significance of the Distribution}

The statistical distribution of both the Telugu treebanks is discussed in detail with their coverage in this section. Here HTTB and HCU-IIIT-H TTB coverage of käraka and nonka raka labels are given one by one. $\mathrm{k} 1$ is $41.9 \%-40.9 \%, \mathrm{k} 1 \mathrm{~s}$ is $4.1 \%-4.7 \%, \mathrm{k} 2$ is $24.9 \%-24.7 \%, \mathrm{k} 2 \mathrm{p}$ is $4.1 \%-0.9 \%, \mathrm{k} 2 \mathrm{~s}$ is $2.0 \%-0.6 \%, \mathrm{k} 3$ is $0.3 \%-0.7 \%, \mathrm{k} 4$ is $5.5 \%-4.7 \%, \mathrm{k} 4 \mathrm{a}$ is $2.1 \%-$ $1.9 \%, \mathrm{k} 5$ is $0.9 \%-1.0 \%, \mathrm{k} 7$ is $2.8 \%-2.4 \%, \mathrm{k} 7 \mathrm{t}$ is $7.6 \%-8.6 \%$, $\mathrm{k} 7 \mathrm{p}$ is $3.7 \%-8.9 \%$, rh is $7.4 \%-3.7 \%$, r6 is $4.1 \%-11.8 \%$, $\mathrm{rt}$ is $2.1 \%-3.3 \%$, vmod is $43.6 \%-20.2 \%$, nmod is $11.3 \%-25.0 \%$, adv is $9.1 \%-14.8 \%$, sent-adv is $2.4 \%-2.2 \%$, ccof is $14.2 \%-10.4 \%$, pof is $2.4 \%-7.9 \%$, ras- $\mathrm{k} 1$ is $3.1 \%-0.7 \%$, ras $-\mathrm{k} 2$ is $0.2 \%-0.1 \%$ in HTTB and HCU-IIIT-H TTB respectively. Here we have considered kāraka and non-kāraka labels separately for calculating the coverage.

In Telugu, there is no overt distinction between $\operatorname{karta}(\mathrm{k} 1)$ and karma $(\mathrm{k} 2)$ at the syntactic level. Because most of the time k1 and $\mathrm{k} 2$ are marked with zero markers (null). Sometimes k1 is also realized with ' $k i$ ', 'ceta' vibhakti markers. In the same way, $\mathrm{k} 2$ is expressed with the 'ni/O' vibhakti marker. The vibhakti 
marker ' $n i$ ' is mandatory for animate nouns and it is optional for inanimate nouns in Telugu. Apart from that semantic information is mandatory to recognize or parse k1 and k2 correctly. In other words, the animate and inanimate distinction should be made in the Treebank data to recognize $\mathrm{k} 1$ and $\mathrm{k} 2$ correctly. Otherwise, there is a chance of recognizing $\mathrm{k} 1$ as $\mathrm{k} 2$ and $\mathrm{k} 2$ as $\mathrm{k} 1$. It may lead to incorrect parsing. By comparing the coverages of the Telugu treebanks, we can say that $65 \%$ of the TTB data is covered by $\mathrm{k} 1$ and $\mathrm{k} 2$ approximately and the remaining $35 \%$ of the TTB data is covered by remaining kāraka labels such as k1s, k2p, k2s, k3, k4, k4a, k5, k7, k7t, $\mathrm{k} 7 \mathrm{p}$, etc. In the case of non-käraka labels, vmod, nmod, adv, and ccof covers more than $60 \%$ of the data, and the remaining $40 \%$ covered by rh, r6, rt, sent-adv, pof, ras-k1, ras-k2 etc. It is observed that k1, k2, vmod, nmod, adv, ccof labels are more important during the creation of the annotated data (Treebank data). The coverage of these four labels is more than $60 \%$. Among k-labels, $\mathrm{k} 3$ has $0.3 \%-0.7 \%$ of the coverage in both the treebanks. The probable reason could be the case marker '-to' ('with') which denotes an instrumental case. But the same case marker '-to' also denotes ras-k1 which means relation for associative with kartā. For example, ravi krṣna tō bajāruku vellădu 'Ravi went to market with Krishna'. Here, 'with Krishna' is marked as ras-k1 but not as k3. Because it does not denote the instrumental case (karaña kāraka). Another reason could be the lack of such constructions in both the Telugu treebanks database. $\mathrm{k} 4$ has $5.5 \%-4.7 \%$ of the coverage in both the Telugu treebanks. The relation $\mathrm{k} 4$ is one of the most ambiguous käraka relations. The dative case marker '-ki' is used to denote the $\mathrm{k} 4$ (sampradāna) relation generally. But it also denotes the hêtu (rh) 'reason' relation (non-kāraka relation). For example, àme domgaki bhayapadimdi 'She feared because of thief'. Here 'because of' is interpreted as hettu (rh) 'reason' but not as sampradāna (k4) 'receiver'. The case 
marker '-ki' is the most ambiguous in Telugu. Because of it, the maximum coverage of $\mathrm{k} 4$ is $5.5 \%$ and a minimum $4.7 \%$ respectively in both TTB's. k5 (source) has 0.9\%-1.0\% coverage only in both the treebanks. The case marker 'numpdi' is used to denote the k5 relation. Syntactic constructions which denote $\mathrm{k} 5$ relation might be less in the database. It is an unambiguous relation in Telugu. Hierarchically $\mathrm{k} 7$ 'viśayādhikarana' is the main kāraka relation and $\mathrm{k} 7 \mathrm{t}$ and $\mathrm{k} 7 \mathrm{p}$ are the sub-tags of $\mathrm{k} 7$. All these three relations are denoted with nouns with space and time (NST). Among these three $(\mathrm{k} 7, \mathrm{k} 7 \mathrm{t}, \mathrm{k} 7 \mathrm{p}), \mathrm{k} 7 \mathrm{p}$ has $3.7 \%-8.9 \%$ coverage, $\mathrm{k} 7 \mathrm{t}$ has $7.6 \%$ $8.6 \%$ coverage, $\mathrm{k} 7$ has $2.8 \%-2.4 \%$ in both the TTB's. NST's (POS tags) have occurred 426, 316 times in both the treebanks. Among all the phrasal categories, NP (Noun Phrase) is the highest frequent phrasal category which has occurred 6223, 7954 times respectively (See Section 4.1 and 4.2). By looking at these frequencies, it is quite natural that some NST's are expected in the natural language. Generally, they are spatial and temporal nouns. In HTTB, $\mathrm{k} 7 \mathrm{t}$ has the highest coverage (7.6\%). In HCU-IIIT-H TTB $\mathrm{k} 7 \mathrm{p}$ has the highest coverage $(8.9 \%)$.

Among non-käraka relations, vmod, nmod, coordination (ccof), sent-adv (Sentential Adverbs) have the highest coverage. Naturally, Paninian Grammar Formalism expresses modifier-modified relations. Among non-kāraka relations, vmod's, nmod's, adverbs, genitives, part of relations (pof) (complex predicates) are large in number. It means that there might be a large number of modifiers that precede the nouns and verbs respectively in the annotated data (both the TTB). They are vmod $43.6 \%-20.2 \%$, nmod $11.7 \%-25.0 \%$, adv 9.1\%$14.8 \%$, r6 $4.1 \%-11.8 \%$, pof $2.4 \%-7.9 \%$ in which most of them are having the highest coverage. The remaining non-käraka relations $\mathrm{rh}$, rt, sent-adv, ras-k1, ras-k2 are having below $8.0 \%$ of the coverage in both TTB's. 
Praveen Gatla

\section{Conclusion}

The present paper is an attempt to compare two Telugu treebanks (HTTB and HCU-IIIT-H TTB). HTTB consists of 2,715 and HCU-IIIT-H TTB consists of 3,222 annotated sentences. Both the treebanks have been created by following DS guidelines which are developed by Bharati, A.; Sharma, D. M.; Husain, S.; Bai, L.; Begam, R. and Sangal, R. (2009). The statistical study has been done at three levels. They are POS, chunk (Phrase), and dependency labels (kāraka and nonkäraka). HTTB sentence length is lesser than HCU-IIIT-H TTB. HTTB has less number of nouns than HCU-IIIT-H TTB at the POS level. Telugu sentences are extracted from Telugu grammars to build HTTB data whereas HCU-IIIT-H TTB data has been extracted from tourism and health domain as a part of IL-IL MT project. In the present study, we found that VM (3,807 times) and $\mathrm{NN}$ (5486 times) are the highest frequent POS categories and QO (11 times), PSP (2 times) are lowest frequent POS categories in HTTB and HCU-IIIT-H TTB respectively. Similarly, NP (7954 and 6223 times) is the highest frequent phrasal category in both the treebanks whereas JJP (7 times), VGINF (6 times) are the lowest frequent phrasal categories in both the treebanks respectively. The major observation is that $65 \%$ of the Telugu treebank data is covered by $\mathrm{k} 1$ and $\mathrm{k} 2$ ( $k \bar{a}$ raka relations). The remaining 35\% is covered by $\mathrm{k} 1 \mathrm{~s}, \mathrm{k} 2 \mathrm{p}, \mathrm{k} 2 \mathrm{~s}, \mathrm{k} 3, \mathrm{k} 4, \mathrm{k} 4 \mathrm{a}, \mathrm{k} 5, \mathrm{k} 7, \mathrm{k} 7 \mathrm{t}, \mathrm{k} 7 \mathrm{p}$. In non-käraka relations, vmod, nmod, adv and ccof has more than $60 \%$ of the coverage. The remaining $40 \%$ is covered by rh, rt, sent-adv, pof, ras-k1, ras-k2 etc. Based on these two statistics, the major coverage is for $\mathrm{k} 1, \mathrm{k} 2$, vmod, nmod, adv, ccof, pof. The coverage of these four labels is more than $60 \%$. This kind of findings will help to make the generalizations based on the statistical frequencies of the treebanks. These generalizations will help the annotator to concentrate on highest frequent labels instead of the lowest frequent labels during the treebank 
validation. In both the TTB's, $k 1, k 2$, vmod, nmod, ccof, sent$a d v$, genitives, pof can be crosschecked or validated for accurate Treebank data. By doing such a kind of statistical study one can know where to spend or concentrate or devout time to improve the treebank data (annotated data). This kind of statistical study is useful to train the human annotators to create the Treebank data. It also helps to boost the accuracy rate of the parsers.

\section{Acknowledgment}

I thank Prof. G. Umamaheshwar Rao who was motivated to create Hyderabad Telugu Treebank. I thank Mr. Y. Vishwanath Naidu and Dr. V. Subrahmanya Madhav Sharma for their valuable discussions to improve the Hyderabad Telugu treebank annotation. I would also like to thank Sneha Nallani, Manish Shrivastava, Dipti Misra Sharma for making the availability of Telugu Treebank (intra-chunk) data for public access under CCLA. Because of it, we could do the statistical study of both the treebanks. I thank the reviewers for their critical comments and suggestions, which helped us to improve the paper.

\section{References}

SAngal, R., Chaitanya V. \& A. Bharati. 1995. Natural Language Processing: A Paninian Perspective. New Delhi: Prentice-Hall.

Bharati, A., R Sangal, D. M. Sharma \& L. Bai. 2006. Anncorra: Annotating Corpora Guidelines for POS and Chunk Annotation for Indian Languages. Hyderabad: IIITTR31. 1-38.

Bharati, A., D. M. Sharma, S. Husain, L. Bai, R. Begam, \& R. SANGAL. 2009. AnnCorra: TreeBanks for Indian Languages, Guidelines for Annotating Hindi TreeBank (version-2.0). Hyderabad: IIIT. 
Bharati, A., M. Gupta, V. YadaV, K. Gali, \& D. M. Sharma. 2009. Simple Parser for Indian Languages in a Dependency Framework. Proceedings of the Third Linguistic Annotation Workshop. 162-165. US: Association for Computational Linguistics.

Bhatt, R., B. Narasimhan, M. Palmer, O. Rambow, D. M. SHARMA \& F. XIA. 2009. A Multi-representational and Multi-layered Treebank for Hindi/Urdu. Proceedings of the Third Linguistic Annotation Workshop (LAW III). 186-189. Singapore: World Scientific Publishing Co Pvt Ltd.

Chatterji, S., P. Sonare, S. SArkar \& D. Roy. 2009. Grammar Driven Rules for Hybrid Bengali Dependency Parsing. Proceedings of ICON09 NLP Tools Contest: Indian Language Dependency Parsing. Hyderabad: IIIT.

De, S., A. DHAR \& U. GARAIN. 2009. Structure Simplification and Demand Satisfaction Approach to Dependency Parsing for Bangla. Proceedings of 6th International Conference on Natural Language Processing (ICON) Tool Contest: Indian Language Dependency Parsing. 25-31. Hyderabad: IIIT.

GADE, R. P. 2014. Dependency Parsing Approaches for Indian Languages: Hindi and Sanskrit. Hyderabad: IIIT doctoral dissertation.

HajiČOVÁ, E. 1998. Prague Dependency Treebank: From Analytic to Tectogrammatical Annotations. Proceedings of 2nd TST. 45-50. Brno, New York: Springer-Verlag Berlin Heidelberg.

KrishnAMURTI, BH. 1991. Studies in Dravidian and General Linguistics: A Festschrift for Bh. Krishnamurti 6. Hyderabad: Osmania University.

KRISHNAMURTI, BH. 2003. The Dravidian Languages. Cambridge: Cambridge University Press.

KrishnamurTi, BH. 2009. Studies in Telugu Linguistics. Hyderabad: C.P. Brown Academy. 
KrishnamuRTI, Bh. \& J. P. L. GWYn. 1985. A Grammar of Modern Telugu. Delhi: OUP.

Krishnamurti, BH. \& P. S. SARMA. 1968. A Basic Course in Modern Telugu. Delhi: Motilal Banarsidass.

Kulkarni, A., S. PoKar \& D. ShuKL. 2010. Designing a Constraint Based Parser for Sanskrit. In International Sanskrit Computational Linguistics Symposium. 70-90. Berlin: Springer.

KULKARNI, A. \& K. RAMAKRISHNAMACHARYULU. 2013. Parsing Sanskrit Texts: Some Relation Specific Issues. In Chaitali Dangarikar \& Malhar Kulkarni (ed.), Proceedings of the 5th International Sanskrit Computational Linguistics Symposium. New Delhi: DK Printworld (P) Ltd.

Marcus, M., B. SANTORINI \& M. A. MarcinkieWicz. 1993. Building a Large Annotated Corpus of English: The Penn Treebank. University of Pennsylvania. Technical Report No. MS-CIS-93-87. Computational Linguistics 19(2). 313-330.

Nallani, S., M. ShrivastaVa \& D. Sharma. 2020. A Fully Expanded Dependency Treebank for Telugu. Proceedings of the WILDRE5- 5th Workshop on Indian Language Data: Resources and Evaluation. Marseille: Language Resources and Evaluation Conference (LREC 2020). 39-44.

Nivre, J., M. C. De MarnefFe, F. Ginter, Y. Goldberg, J. Hajic, C. D. Manning, R. McDonald, S. Petrov, S. Pyysalo, N. SilveIRA \& R. TSARFATY. 2016. Universal Dependencies v1: A Multilingual Treebank Collection. Proceedings of the Tenth International Conference on Language Resources and Evaluation (LREC'16). 16591666.

PRAKASAM, V. 2018. Aadhunika Telugu Vennelakanti Vyaakaranam. Hyderabad: Nirrvitha Publishing.

PraveEN, G. 2019. Developing a Parser for Telugu. Hyderabad: HCU doctoral dissertation. 
RAJYARAMA, K. \& A. SRINIVAS. 2015a. Issues in Developing a Dependency Parser for Telugu: A Linguistic Account. BHASHAA: An International Journal of Telugu Linguistics. 93-101. Magazine-4. Hyderabad: Telugu Linguists' Forum.

RAJYARAMA, K. \& A. SRINIVAS. 2015b. Yantranuvadamlo Ani. BHASHAA: An International Journal of Telugu Linguistics. 35-45. Magazine-1. Hyderabad: Telugu Linguists' Forum.

Rama, T. \& S. VajJala. 2018. A Dependency Treebank for Telugu. Proceedings of the 16th International Workshop on Treebanks and Linguistic Theories (TLT16). 119-128. Prague: Czech Republic.

RAMARAO, C. 1975. Telugu Vakyam. Hyderabad: Andhra Pradesh Sahitya Academy.

RamaraO, C. 2002. Quest of Subject in Telugu. In Swarajya Lakshmi (ed.), Case for Language Studies 30. 153-156. Hyderabad: Book Links Corporation.

Shailaja, N. 2009. Parser for Simple Sanskrit Sentences. Hyderabad: HCU M.Phil dissertation.

SRINIVAS, A. 2012. Telugu Bhasha-Vyakaranam. Hyderabad: Akruthi Offset Printers.

SRINIVAS, A. \& K. RAJYARAMA. 2015. Hindi-Telugu Yantranuvadamlo Yokka (sheșa șașṭi): Alopa Sutralu. BHAASHA: International Journal of Telugu Linguistics 4. 58-67. Hyderabad: Telugu Linguists' Forum.

Subbarao, K. V. 2012. South Asian Languages: A Syntactic Typology. Cambridge: CUP.

SUBRAHMANYAM, P. S. 1984. Adhunika Bashashastra Sidhantalu. Hyderabad: Andhra Pradesh Sahitya Academy. TESNiÈRE, L. 1959. Eléments de Syntaxe Structurale. (Timothy Osborne \& Sylvain Kahane, Trans.). Amsterdam: John Benjamins.

Umamaheshwar RaO, G., R. KopPaKa, \& S. AdDANKi. 2012. Dative Case in Telugu: A Parsing Perspective. Proceedings 
A Statistical Study of Telugu Treebanks

of the Workshop on Machine Translation and Parsing in Indian Languages. 123-132. Mumbai: COLING.

Usha RANI, A. 1980. Relativization in Telugu. Hyderabad: Osmania University doctoral dissertation.

Vempaty, C., V. Naidu, S. Husain, R. Kiran, L. Bai, D. M. Sharma \& R. SANGAL. 2010. Issues in Analyzing Telugu Sentences Towards Building a Telugu Treebank. International Conference on Intelligent Text Processing and Computational Linguistics. 50-59. New York: Springer.

\section{Cite This Work:}

Gatla, PraveEn. 2021. A Statistical Study of Telugu Treebanks. Translation Today, Vol. 15(1). 145-167.

DOI:10.46623/tt/2021.15.1.ar6 